\title{
Analysis of Transmission Characteristics of Tilted Long Period Fiber Gratings With Full Vector Complex Coupled Mode Theory
}

\author{
Qi LI ${ }^{1}$, Fengping YAN ${ }^{1}$, Peng LIU $^{2}$, Wanjing PENG ${ }^{1}$, Guolu YIN ${ }^{1}$, and Ting FENG ${ }^{1}$ \\ ${ }^{1}$ Key Lab of All Optical Network and Advanced Telecommunication, Institute of Lightwave Technology, Beijing Jiaotong \\ University, Beijing, 100044, China \\ ${ }_{2}^{2}$ Physics Department of Xingtai College, Xingtai, 054001, China \\ *Corresponding author: Fengping YAN_E-mail: fpyan@bjtu.edu.cn
}

\begin{abstract}
Based on the full vector complex coupled mode theory, a detailed analysis is made on the transmission spectrum characteristics of tilted long period fiber gratings. New transmission peaks are observed, which are located beside the long wavelength side of each transmission peak in the transmission spectrum of normal long period fiber gratings. The emerging transmission peaks are quite sensitive to both the grating tilted angle and the surrounding refractive index, and the corresponding relationship is discussed. Furthermore, a novel sensing characteristic is investigated about the tilted long period fiber gratings, which is related to the transmission resonant wavelength and peak amplitude.
\end{abstract}

Keywords: Optical fiber gratings, fiber optic sensors, tilted long period fiber gratings, numerical analysis

\section{Introduction}

As important passive optical devices, fiber Bragg gratings (FBGs) and long period fiber gratings (LPFGs) are widely used in the fiber optical communication and sensing fields [1-6]. Correspondingly, many investigations are made on them [7-13]. Recently, the titled fiber gratings, especially the tilted fiber Bragg gratings (TFBGs) with the small grating period have attracted tremendous attention due to their applications in the sensing area [14-22].

Similar to the LPFGs, tilted long period fiber gratings (TLPFGs) are also transmission gratings without back reflectance. But the TLPFGs show different characteristics compared with the LPFGs for their tilted grating plane. There are new emerged transmission peaks beside the long wavelength side of each transmission peak in the transmission spectrum. The new transmission peaks, which are caused by the tilted angle, have new feature different from that of LPFGs. The new characteristics of the TLPFGs bring a new dimension for the design of optical fiber sensors. In this paper, an analysis is made on transmission characteristics of TLPFGs with the full vector complex coupled mode theory (FV-CCMT). This theoretical model can be used to analyze the tilted fiber gratings under different scenarios effectively.

Only the coupling between vector fiber modes with identical azimuthal orders $\mu$ (usually $\mu=1$ ) is allowed in non-tilted fiber gratings [9]. However,

Received: 9 October 2011 / Revised version: 29 December 2011

(C) The Author(s) 2012.This article is published with open access at Springerlink.com 
when there is a tilt of the grating plane, this rule is broken; thus, vector fiber modes with dissimilar azimuthal orders can couple with each other. And the resonance strength is also enhanced [3, 7, 8, 14]. The coupling between the core mode and the cladding modes with the azimuthal order $(\mu \neq 1)$ will induce new emerging transmission peak which shows new characteristics. In this paper, the TLPFGs are investigated theoretically through the transmission spectrum. The influence of the tilted angle $(\theta)$ and the refractive index of the surrounding medium $\left(n_{\mathrm{s}}\right)$ are discussed.

\section{Complex coupled mode equations for tilted long period fiber gratings}

Figure 1 is the schematic diagram of the TLPFG.
The TLPFGs are written in the single mode fiber and fabricated by the ultraviolet(UV)-exposure method. The oscillation of the electric field of p-mode is in parallel with the $x$ axis in the $x-z$ plane, and s-mode is perpendicular to the $x-z$ plane. The tilted angle $(\theta)$ of the TLPFGs in this paper satisfies $\theta>45^{\circ}$. The co-propagating coupling occurs between radiation mode and propagating mode when the tilted angle $(\theta)$ satisfies $\theta>45^{\circ}$, which is shown in Fig. 2. When the vectors satisfy the phase matching condition: $\mathbf{K}_{\mathrm{co}}=\mathbf{K}_{\mathrm{r}}+\mathbf{K}_{\mathrm{g}}$, the strongest interaction occurs. The index variation along the longitudinal axis of the fiber core can be written as [8]

$$
\Delta n\left(z^{\prime}\right)=\bar{v}\left(z^{\prime}\right)+2 \bar{\rho}\left(z^{\prime}\right) \cos \left[2 K_{g} z^{\prime}+\bar{\phi}\left(z^{\prime}\right)\right]
$$
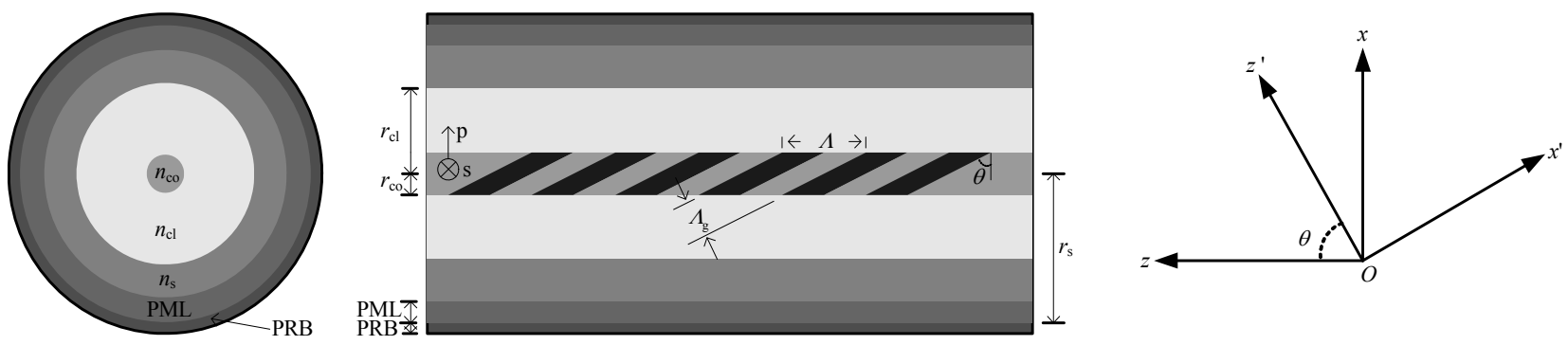

Fig. 1 Diagram of a TLPFG written in a fiber with the three-layer structure.

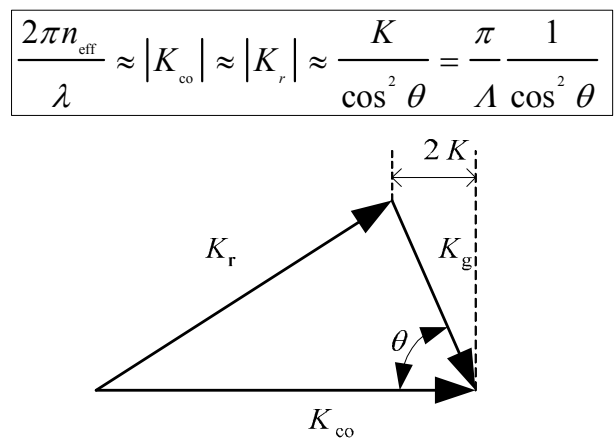

Fig. 2 Phase matching condition in TLPFGs $\left(\theta>45^{\circ}\right)$.

where $\bar{v}\left(z^{\prime}\right)$ and $\bar{\rho}\left(z^{\prime}\right)$ describe the slowly varying DC perturbation spatially averaged over a grating period, $K_{\mathrm{g}}$ means the nominal wave number of the grating, $\bar{\phi}\left(z^{\prime}\right)$ is the position-dependent chirp, $n\left(z^{\prime}\right)$ is required only at points in the fiber core. For the length of the grating is much larger than the diameter of the fiber core $(z>>x)$, it is permissible to put $z^{\prime}=x \sin (\theta)+z \cos (\theta) \approx z \cos (\theta)$ in the slowly varying functions. As shown in Figs. 1 and 2, the relation between $K_{\mathrm{g}}$ and $K$ satisfies $K_{g}=K / \cos (\theta)$. The index variation along the longitudinal axis of the fiber core $\Delta n$ can be rewritten as

$\Delta n(z)=v(z)+2 \rho(z) \cos \{2 K[z+x \tan (\theta)]+\phi(z)\}$.

In terms of the normalized power fields of the p-mode and s-mode, the transverse fields of the TLPFGs can be expanded as

$$
\left\{\begin{array}{l}
E_{t}\left(r_{t}, z\right)=\sum_{p= \pm} \sum_{m}\left[a_{m}^{p}(z)+b_{m}^{p}(z)\right] e_{m}^{p}\left(r_{t}\right) \\
H_{t}\left(r_{t}, z\right)=\sum_{p= \pm} \sum_{m}\left[a_{m}^{p}(z)-b_{m}^{p}(z)\right] h_{m}^{p}\left(r_{t}\right)
\end{array}\right.
$$

where $e_{m}\left(r_{t}\right)$ and $h_{m}\left(r_{t}\right)$ are the normalized transverse electric field and magnetic field, $a_{m}(z)$ and $b_{m}(z)$ are the forward amplitude and backward amplitude of $m$-th mode, "+" and "-" are the p-mode and s-mode, respectively. By inserting (3) into Maxwell's functions, considering the orthogonality of the modes (p-mode and s-mode), the amplitude 
equations for $a_{m}^{p}(z)$ and $b_{m}^{p}(z)$ can be derived as [11]

$$
\left\{\begin{array}{l}
\frac{\partial a_{m}^{p}}{\partial z}+\mathrm{j} \beta_{m}^{p} a_{m}^{p}=-\mathrm{j} \sum_{q= \pm} \sum_{n}\left(\kappa_{m n}^{p q} a_{n}^{q}+\chi_{m n}^{p q} b_{n}^{q}\right) \\
\frac{\partial b_{m}^{p}}{\partial z}+\mathrm{j} \beta_{m}^{p} b_{m}^{p}=+\mathrm{j} \sum_{q= \pm} \sum_{n}\left(\chi_{m n}^{p q} a_{n}^{q}+\kappa_{m n}^{p q} b_{n}^{q}\right)
\end{array}\right.
$$

where $\kappa_{m n}^{p q}$ and $\chi_{m n}^{p q}$ are the coupling coefficients between different modes. Due to the tilt angle of the gratings, the p-mode and s-mode can not couple with each other. The TLPFGs are transmission types with the grating tilt angle $\theta>45^{\circ}$, and the co-propagating coupling occurs. The strongest interaction of an individual resonance occurs when the wave vectors satisfy the phase matching condition:

$$
\Delta=\frac{2 \pi}{\lambda_{C}} \operatorname{Re}\left(n_{\text {eff }}^{\mathrm{co}}-n_{\text {eff }}^{\mathrm{cl}}\right)-\frac{2 \pi}{\Lambda}=\bar{\beta}_{\mathrm{co}}-\bar{\beta}_{\mathrm{cl}}-\frac{2 \pi}{\Lambda} \approx 0 .
$$

The period of the fiber grating is uniform in this paper. The amplitude equation ultimately can be derived as

$$
\left\{\begin{array}{l}
\frac{d a_{1}}{d z}=-\mathrm{j} \sum_{n \neq 1} K_{1 n}^{-} \rho(z) a_{n}(z) \exp \left[-\mathrm{j}\left(\bar{\beta}_{n}-\bar{\beta}_{1}\right) z\right]+\mathrm{j} k_{1}(z) a_{1}(z)-\mathrm{j} \bar{\beta}_{1} a_{1}(z) \\
\frac{d a_{n}}{d z}=-\mathrm{j} K_{n 1}^{+} \rho(z) a_{1}(z) \exp \left[-\mathrm{j}\left(\bar{\beta}_{n}-\bar{\beta}_{1}\right) z\right]-\mathrm{j} k_{n}(z) a_{n}(z)-\mathrm{j} \bar{\beta}_{n} a_{n}(z)
\end{array}\right.
$$

where $\bar{\beta}_{n}=\operatorname{Re}\left(\beta_{n}\right)$, and the parameters $K_{1 n}^{-}, K_{n 1}^{+}$, $k_{1}(z), k_{n}(z)$ can refer to [11].

\section{Numerical results and discussion}

In this section, the transmission spectrum of TLPFGs is calculated by the FV-CCMT. The related parameters of the TLPFGs are shown in Fig. 1. The TLPFGs are written in a single mode fiber with $r_{\mathrm{co}}=3.5 \mu \mathrm{m}, \quad r_{\mathrm{cl}}=50 \mu \mathrm{m}, \quad r_{\mathrm{s}}=62.5 \mu \mathrm{m}, \quad n_{\mathrm{cl}}=1.465$, $\Delta=\left(n_{\mathrm{co}}^{2}-n_{\mathrm{cl}}^{2}\right) /\left(2 n_{\mathrm{co}}^{2}\right)=0.34 \% \quad, \quad n_{\mathrm{s}}=1.0 \quad$ and $2 \gamma=3.8 \times 10^{-4}$. The mesh size is $\Delta r=0.08 \mu \mathrm{m}$. The grating period is fixed as $\Lambda=340 \mu \mathrm{m}$, and the length $(L)$ of the gratings is $5 \mathrm{~cm}$.

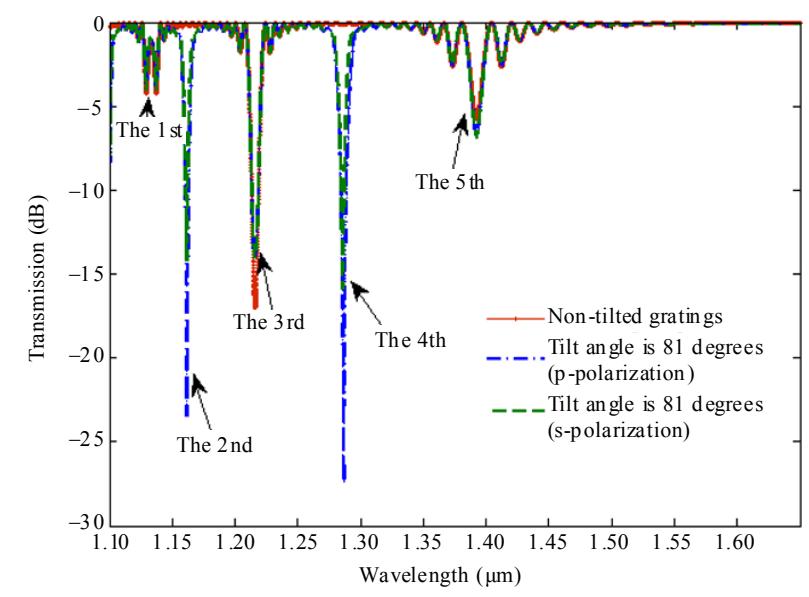

Fig. 3 Transmission spectrum of the LPFGs and TLPFGs.

Since the index modulation section plane is no longer perpendicular to the fiber axis, the s-polarized light and p-polarized light experience different index changes along the fiber, which will result in difference transmission characteristics. Figure 3 shows the transmission spectrum of the non-tilted long period fiber gratings and tilt long period fiber gratings, respectively. There are mainly five peaks in the transmission spectrum, which are labeled as the 1st to 5th along with an increase in the wavelength as shown in Fig. 3. When the gratings are non-tilted gratings, there are only three peaks in the transmission spectrum i.e. the 1st, 3rd and 5th peaks. If the gratings are blazed, new peaks will emerge i.e. the 2 nd and 4 th peaks. Similar to the TFBGs, the new emerging transmission peaks in TLPFGs are induced by the coupling to the vector fiber modes with the azimuthally order $m \neq 1$ [8].

\subsection{Influence of the grating tilted angle}

The only difference between TLPFGs and LPFGs is the tilt angle of the grating plane, so it is of interest to find the effect of the tilt angle through the transmission spectrum. In this section, the transmission spectrum characteristics of TLPFGs will be analyzed, and the influence of grating tilted angle will be discussed. The 1st, 3rd and the 5th resonances are mainly due to the coupling between the fundamental mode and the $m=1$ cladding mode. However, the 2nd and the 4th resonances are mainly due to the coupling from the fundamental mode to the $m \neq 1$ cladding modes. The former kind of 
coupling exists in both tilted and non-tilted LPFGs. The latter kind of coupling only occurs in TLPFGs. In this paper, the investigation just focuses on the transmission peaks from the 2 nd to 5 th, which are deeper than $5 \mathrm{~dB}$ as shown in Fig. 3. The outer-cladding of the TLPFGs are assumed to be air with a refractive index of 1 . The perfect matched layer (PML) is also not utilized.

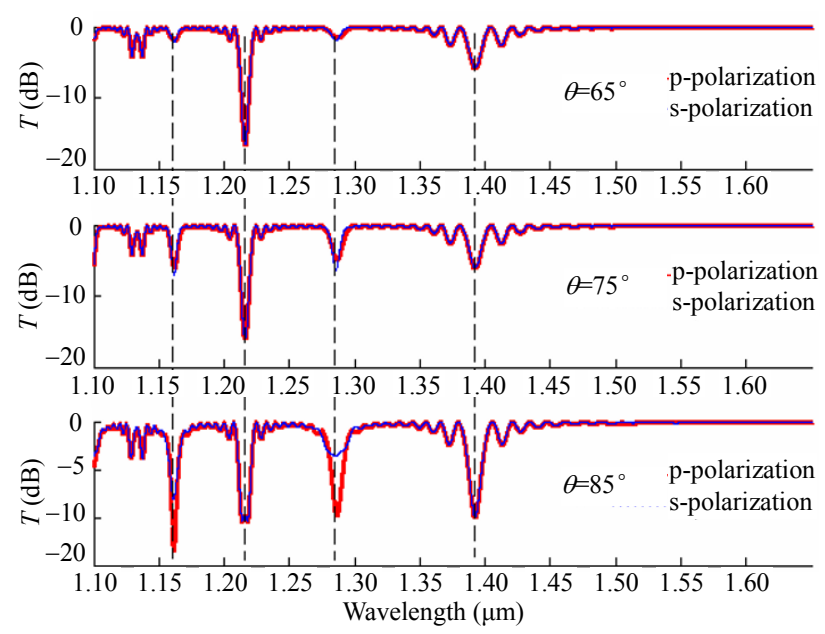

Fig. 4 Transmission spectrum evolution of TLPFGs with an increase in the tilt angle $\left(n_{\mathrm{s}}=1\right)$.

For further comparison, the transmission spectrum evolution of TLPFGs with an increase in the tilt angle is shown in Fig. 4. The amplitudes of the four transmission peaks vary with the tilt angle. According to $\lambda_{D}=\operatorname{Re}\left(n_{\text {eff }}^{\text {co }}-n_{\text {eff }}^{\text {cl }}\right) \cdot \Lambda$, the resonant wavelengths $\left(\lambda_{D}\right)$ of the transmission peaks are virtually constant when the grating period $(\Lambda)$ is fixed. There are about 1-nm gap between the two (pand s-) polarization light of the 2nd and 4th transmission peaks. On the contrary, the different polarization light of the 3rd and 5th transmission peaks overlaps with each other. With an increase in the tilt angle, these cases remain unchanged. The corresponding minimum transmission peaks are shown in Figs. 5(a), 5(b), 5(c), and 5(d), respectively.

It is clear that the 2 nd and 4 th peaks possess the complex change versus the tilt angle, which only occurs in TLPFGs. By contrast, the 3rd and 5th peaks have simple change from these figures. Figure 5(a) displays the 2nd transmission peak amplitude evolution and the difference of the two (p- and s-) polarization light with respect to the tilt angle. The amplitudes of the transmission peaks of the two polarization light separate from each other at different levels, except ranging from 65 degrees to 75 degrees and 80 degrees, 86 degrees, 87 degrees, 88.2 degrees, 88.9 degrees. The variations of the amplitudes of the transmission peaks of the two polarization light are very slow ranging from 65 degrees to 75 degrees and the amplitudes vibrate very rapidly at different levels for the rest of the angles. The maximum absolute amplitude difference value of the two polarization light transmission peaks is $30.2 \mathrm{~dB}$ at about 80.5 degrees.

The 4th transmission peak amplitude evolution is parallel to the 2nd transmission peak, as shown in Fig. 5(c). The absolute difference value of the transmission peak amplitudes of the two polarization light equals to zero at the tilt angle of 80.6 degrees, 86.2 degrees, 87.6 degrees, 88.9 degrees besides ranging from 65 degrees to 75 degrees. And the maximum absolute difference value is $43 \mathrm{~dB}$ at about 81.3 degrees.

As shown in Figs. 5(b) and 5(d), the variation tendency of the amplitude of the two (p- and s-) polarization light is practically identical versus the tilt angle from 65 degrees to 89 degrees. The evolution of the resonance strength of the $3 \mathrm{rd}$ and 5 th transmission peaks is opposite to each other. The resonance strength of the $3 \mathrm{rd}$ peak becomes weaker along with an increase in the tilt angle, as shown in Fig. 5(b). On the contrary, the resonance strength of the 5th peak becomes stronger along with an increase in the tilt angle from 65 degrees to 87 degrees, as shown in Fig. 5(d).

The variations of the two (p- and s-) polarization light of the 2nd and 4th transmission peaks are different along with the tilted angle, and this characteristic has good application prospects in the fiber twist sensor [16].

The variations of the two (p- and s-) polarization light of the 2nd and 4th transmission peaks are complex. The two peaks are induced by the coupling 


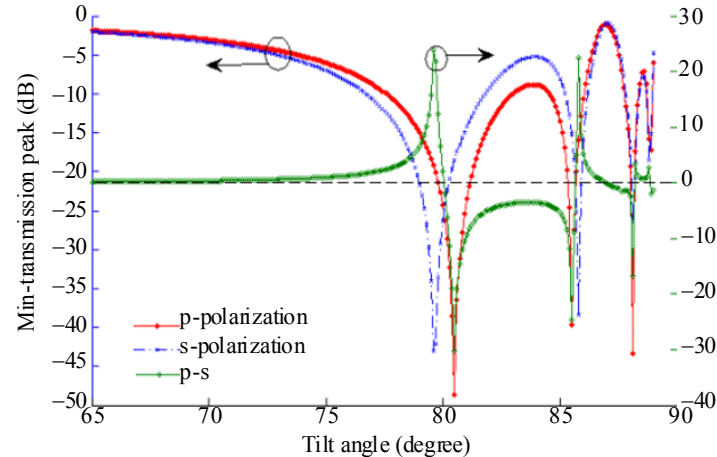

(a)

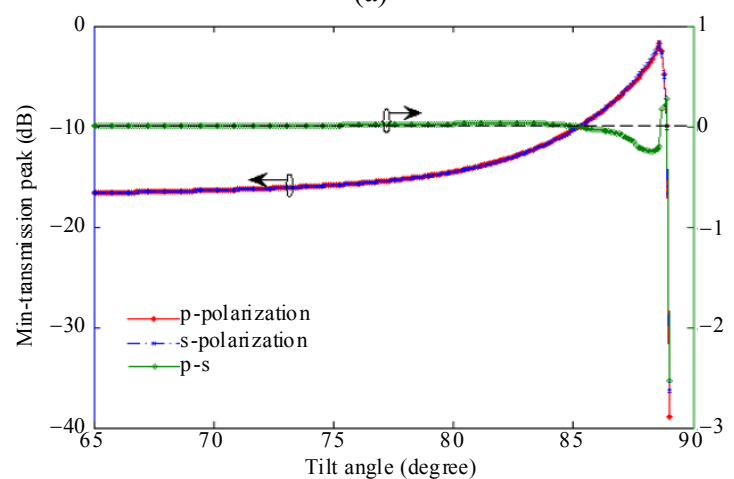

(b)

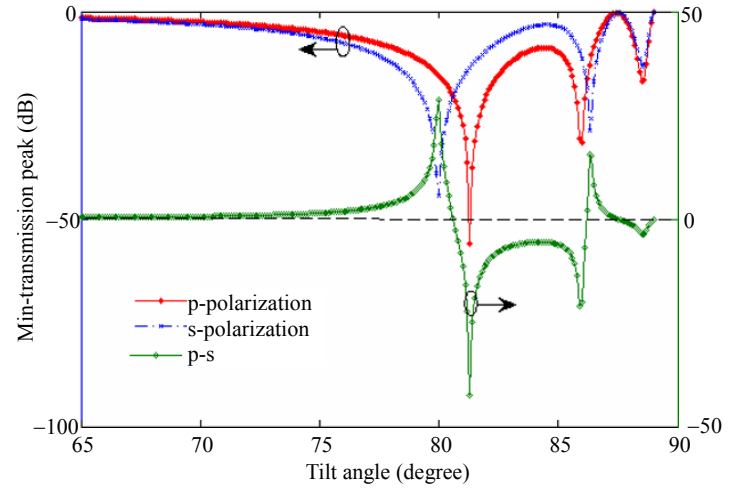

(c)

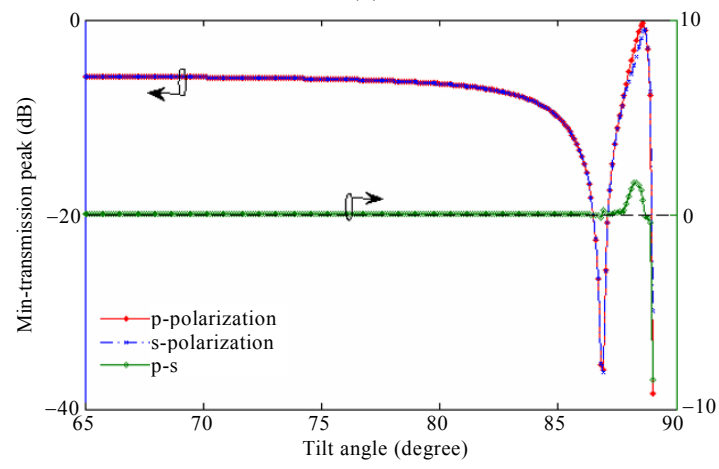

(d)

Fig. 5 Transmission spectrum evolution of TLPFGs with an increase in the tilt angle: the amplitudes of the transmission peaks-(a) the 2nd, (b) the 3rd, (c) the 4th, and (d) the 5th. between core mode and non-circular-symmetrical caldding modes. Along with an increase in the tilt angle, the coupling between a core mode and cladding modes $(m>1)$ becomes stronger and starts to be weaker after the coupling gets to the strongest. Furthermore, the coupling between a core mode and higher-order (larger mode number $m$ ) cladding modes becomes stronger again and starts to be weaker again after the coupling gets to the strongest. The variations of the two (p- and s-) polarizationlight of the 2nd and 4th transmission peaks are different along with tilted angle, and this characteristic has good application prospects in the fiber twist sensor [16].

\subsection{Influence of the refractive index of the surrounding medium}

The outer-cladding of TLPFGs was assumed to be air in the Section 3.1. The spectral characteristics of the TLPFGs and the influence of the grating tilted angle have been analyzed. In this section, the influence of the refractive index $\left(n_{\mathrm{s}}\right)$ of the surrounding medium will be discussed.

Figure 6 shows that all the resonant wavelengths of the predominant four transmission peaks shift to the shorter wavelengths with an increase in $n_{\mathrm{s}}$. However, the evolution of the amplitude of each peak versus $n_{\mathrm{s}}$ is independent. There is about $1-\mathrm{nm}$ gap between p- and s- polarization light of the 2 nd and 4th transmission peaks, on the contrary, the different polarization light of the 3rd and 5th transmission peaks overlaps with each other. The resonant wavelength of the two (p- and $s-$ ) polarization light of each transmission peak vary identically with an increase in $n_{s}$. These two different polarization light almost stays at the constant level along with an increase in $n_{\mathrm{s}}$ from 1.0 to 1.3 and goes into slow evolution with $n_{\mathrm{s}}$ ranging from 1.3 to 1.45 and varies very rapidly when $n_{\mathrm{s}}$ is larger than 1.45 in Figs. 7(a), 7(b), 7(c), and $7(\mathrm{~d})$.

As shown in Figs. 7(b) and 7(d), the variation tendency of the amplitudes of the two (p- and s-) 

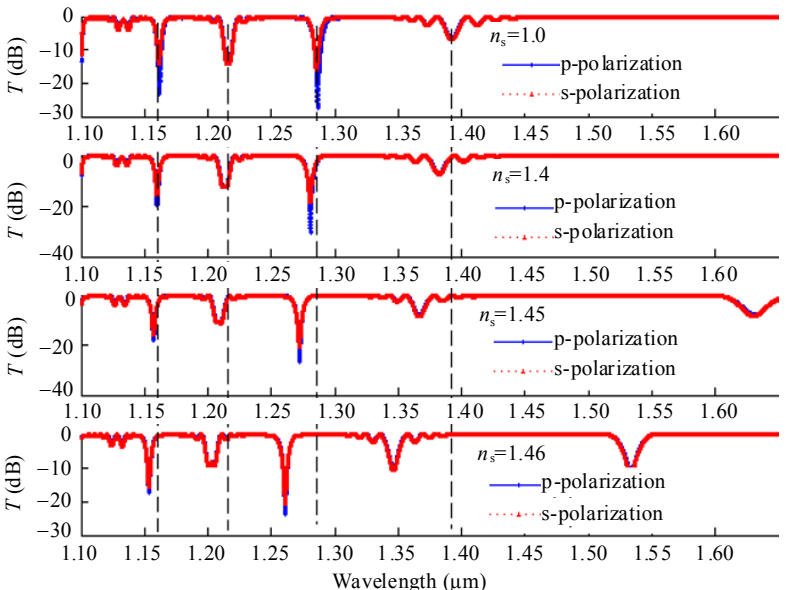

Fig. 6 Spectrum of the transmission versus the refractive index of the surrounding medium $\left(n_{\mathrm{s}}\right)$.

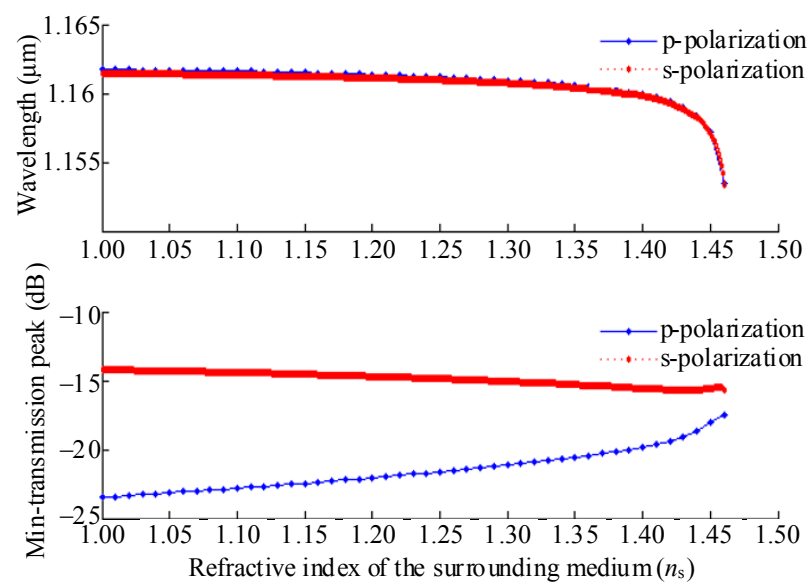

(a)
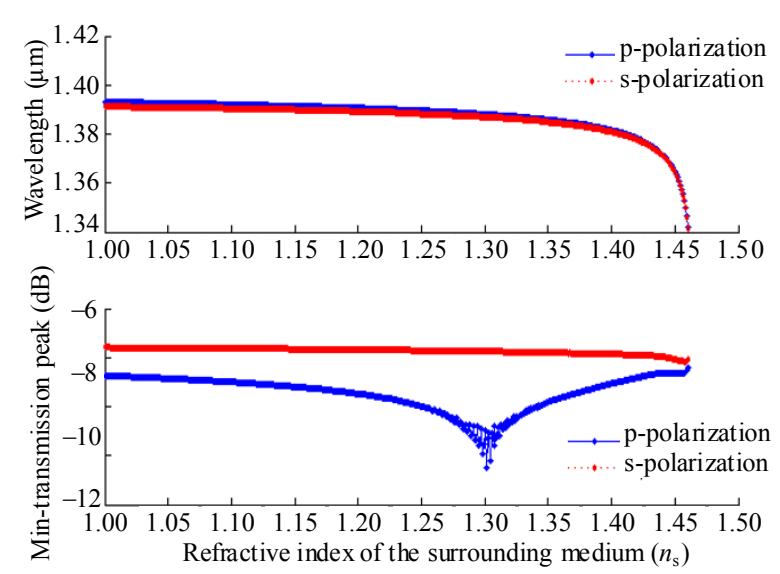

(c) polarization light is practically identical versus $n_{\mathrm{s}}$ from 1.0 to 1.46. The 3rd transmission peak amplitude increases from $-14 \mathrm{~dB}$ to $-9.5 \mathrm{~dB}$. On the contrary, the amplitude of the 5 th transmission peak decreases from $-6.8 \mathrm{~dB}$ to $-10.9 \mathrm{~dB}$ with an increase in $n_{\mathrm{s}}$ from 1.0 to 1.46 .

As shown in Fig. 7(a), the amplitudes of the two (p- and s-) polarization light of the 2 nd peak are disparate with $n_{\mathrm{s}}(1.0-1.46)$. The amplitude of the s-polarization light is almost unchanged. By contrast, the amplitude of p-polarization light increases from $-23 \mathrm{~dB}$ to $-17.4 \mathrm{~dB}$ with $n_{\mathrm{s}}$ ranging from 1.0 to 1.46 .
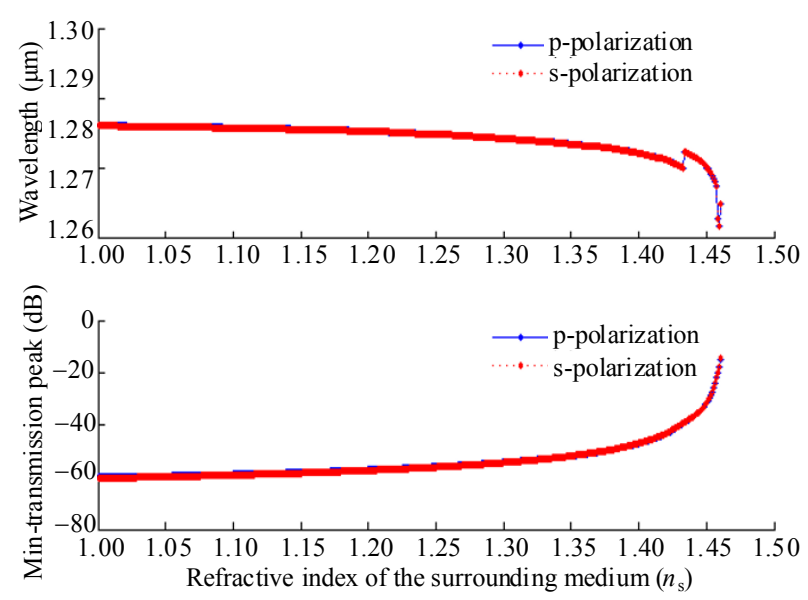

(b)
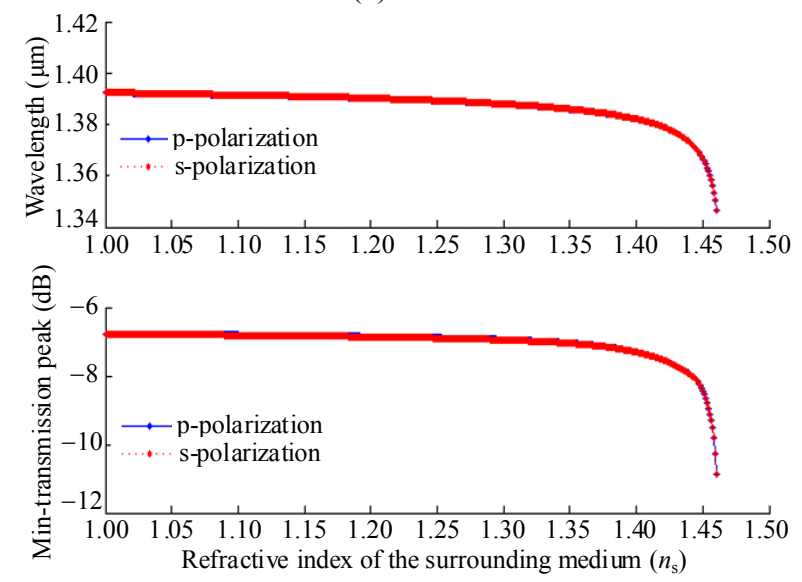

(d)

Fig. 7 Transmission spectrum evolution of TLPFGs with the refractive index of the surrounding medium $\left(n_{\mathrm{s}}\right)$ : the resonant wavelengths and amplitudes of transmission peaks-(a) the 2nd, (b) the 3rd, (c) the 4th, and (d) the 5th.

The amplitudes of the two (p- and s-) polarization light of the 4 th peak are disparate with $n_{\mathrm{s}}$ ranging from 1.0 to 1.46 , which is shown in
Fig. 7(c). The amplitude of the s-polarization light decreases from $-15.9 \mathrm{~dB}$ to $-21.7 \mathrm{~dB}$, but by contrast the p-polarization light has a complex evolution 
versus an increase in $n_{\mathrm{s}}$. The amplitude of the p-polarization light has a rapid fluctuation with an increase in $n_{\mathrm{s}}$ from 1.15 to 1.4 , especially from 1.25 to 1.35 . The minimum transmission amplitude of the p-polarization light even reaches $-64.8 \mathrm{~dB}$ when the refractive index is about 1.3. The whole evolution range of the amplitude of p-polarization light reaches about $41 \mathrm{~dB}$.

The novel characteristics of the 2nd and 4th peaks distinguish TLPFGs from normal LPFGs. Thanks to the 2nd and 4th peaks induced by the coupling between a core mode and cladding modes $(m>1)$, the sensitivity of higher-order cladding modes $(m>1)$ to the refractive index of the surrounding medium is more sensitive than that of the cladding mode with the azimuthal order $m=1$, and the sensitivities of the two peaks to the refractive index of the surrounding medium are remarkable. The surrounding medium refractive index sensitivity of the TLPFGs has good application prospects in the optical fiber refractive index sensing field, especially the brilliant refractive index sensitivity around 1.3 of the 4th transmission peak.

\section{Conclusions}

New transmission peaks are observed for the first time, which are located beside the long wavelength side of each transmission peak in the transmission spectrum. The new transmission peaks are sensitive to the polarization and refractive index of the surrounding medium, which have good application prospects in the optical fiber sensing field.

The min-transmission amplitude of each peak varies with the tilt angle, however, the resonant wavelength of each peak remains unchanged versus an increase in the tilt angle when the grating period is fixed. According to the numerical analysis, there are several amplitude intersections, and the maximum amplitude gaps are about $30.2 \mathrm{~dB}$ and 43 $\mathrm{dB}$ between the $\mathrm{p}$ - and s-polarization light of the two new emerging transmission peaks along with the tilted angle. So these characteristics can be used in the optical fiber twist sensor and should be used in polarizer and polarization suppression.

The brilliant sensitivity of TLPFGs to the refractive index of the surrounding medium due to the new emerging transmission peaks is beyond that of the normal LPFGs, especially when the refractive index is around 1.3. New type optical fiber refractive index sensor could be designed according to the novel characteristic of TLPFGs.

\section{Acknowledgment}

This work is supported by the National Natural Science Foundation of China (No. 61077069) and the Fundamental Research Funds for the Central Universities (Beijing Jiaotong University No. 2011YJS003).

Open Access This article is distributed under the terms of the Creative Commons Attribution License which permits any use, distribution, and reproduction in any medium, provided the original author(s) and source are credited.

\section{References}

[1] V. Bhatia and A. M. Vengsarkkar, "Optical fiber long-period grating sensors," Optics Letters, vol. 21, no. 9, pp. 692-694, 1996.

[2] X. W. Shu, L. Zhang, and I. Bennion, "Sensitivity characteristics of long-period fiber grating," Journal of Lightwave Technology, vol. 20, ,no. 2, pp. 255-266, 2002.

[3] K. O. Hill, B. Malo, K. A. Vineberg, F. Bilodeau, D. C. Johnson, and I. Skinner, "Efficient mode conversion in telecommunication fiber using externally written gratings," Electronics Letters, vol. 26, no. 16, pp. 1270-1272, 1990.

[4] G. B. Hocker, "Fiber-optic sensing of pressure and temperature," Applied Optics, vol. 18, no. 9, pp. 1445-1448, 1979.

[5] M. G. Xu, L. Peekie, Y. T. Chow, and J. P. Dakin, "Optical in-fiber grating high pressure sensor," Electronics Letters, vol. 29, no. 4, pp. 398-399, 1993.

[6] M. G. Xu, H. Geiger, and J. P. Dakin, "Fiber grating 
pressure sensor with enhance sensitivity using a glass-bubble housing," Electronics Letters, vol. 32, no. 2, pp. 128-139, 1996.

[7] T. Erdogan, "Fiber grating spectra," Journal of Lightwave Technology, vol. 15, no. 8, pp. 1277-1294, 1997.

[8] T. Erdogan and J. E. Sipe, "Tilted fiber phase gratings," Journal of the Optical Society of America A, vol. 13, no. 2, pp. 296-313, 1996.

[9] T. Erdogan, "Cladding-mode resonances in short and long period fiber grating filters," Journal of the Optical Society of America A, vol. 14, no. 8, pp. 1760-1773, 1997.

[10] Y. C. Lu, W. P. Huang, and S. S. Jian, "Polarization sensitivities of demodulation techniques for tilted fiber Bragg grating refractometer," in Proc. SPIE (Asia Communications and Photonics Conference and Exhibition), vol. 7630, pp. 76300U , 2009.

[11] Y. C. Lu, W. P. Huang, and S. S. Jian, "Full vector complex coupled mode theory for tilted fiber gratings," Optics Express, vol. 18, no. 2, pp. 713-725, 2010.

[12] Y. C. Lu, L. Yang, W. P. Huang, and S. S. Jian, "Improved full-vector finite-difference complex mode solver for optical waveguides of circular symmetry," Journal of Lightwave Technology, vol. 26, no. 13, pp. 1868-1876, 2008.

[13] Y. C. Lu, L. Yang, W. P. Huang, and S. S. Jian, "Unified approach for coupling to cladding and radiation modes in fiber Bragg and long-period gratings," Journal of Lightwave Technology, vol. 27, no. 11, pp. 1461-1468, 2009.

[14] R. Kashyap, R. Wyatt, and R. Campbell, "Wideband gain flattened erbium fiber amplifier using a photosensitive fiber blazed grating," Electronics Letters, vol. 29, no. 2, pp. 154-156, 1993.
[15] L. Y. Shao, L. Y. Xiong, C. K. Chen, A. Laronche, and J. Albert, "Directional bend sensor based on re-grown tilted fiber Bragg grating," Journal of Lightwave Technology, vol. 28, no. 18, pp. 2681-2687, 2010.

[16] X. Chen, K. Zhou, L. Zhang, and I. Bennion, "In-fiber twist sensor based on a fiber Bragg grating with $81^{\circ}$ tilted structure," IEEE Photonics Technology Letters, vol. 18, no. 24, pp. 2596-2598, 2006.

[17] E. Chehura, S. W. James, and R. P. Tatam, "Temperature and strain discrimination using a single tilted fiber Bragg grating," Optics Communications, vol. 275, no. 2, pp. 344-347, 2007.

[18] T. Guo, H. Y. Tam, P. A. Krug, and J. Albert, "Reflective tilted fiber Bragg grating refractometer based on strong cladding to core recoupling," Optics Express, vol. 17, no. 7, pp. 5736-5742, 2009.

[19] R. Kashyap, R. Wyatt, and R. J. Campbell, "Wideband gain flattened erbium fiber amplifier using a photosensitive fiber blazed grating," Electronics Letters, vol. 29, no. 2, pp. 154-156, 1993.

[20] Y. C. Lu, R. Geng, C. C. Wang, F. Zhang, C. Liu, T. G. Ning, and S. S. Jian, "Polarization effects in tilted fiber Bragg grating refractometers," Journal of Lightwave Technology, vol. 28, no. 11, pp. 1677-1684, 2009.

[21] R. Wu, Y. Q. Liu, J. Zou, N. Chen, F. F. Pang, and T. Y. Wang, "Fabrication of tilted long-period fiber gratings by $\mathrm{CO}_{2}$ laser," in Proc. SPIE, vol. 8307, pp. 83072D, 2011.

[22] Y. P. Wang, "Review of long period fiber gratings written by $\mathrm{CO}_{2}$ laser," Journal of Applied Physics, vol. 108, no.8, pp. 081101-1-081101-18, 2010. 